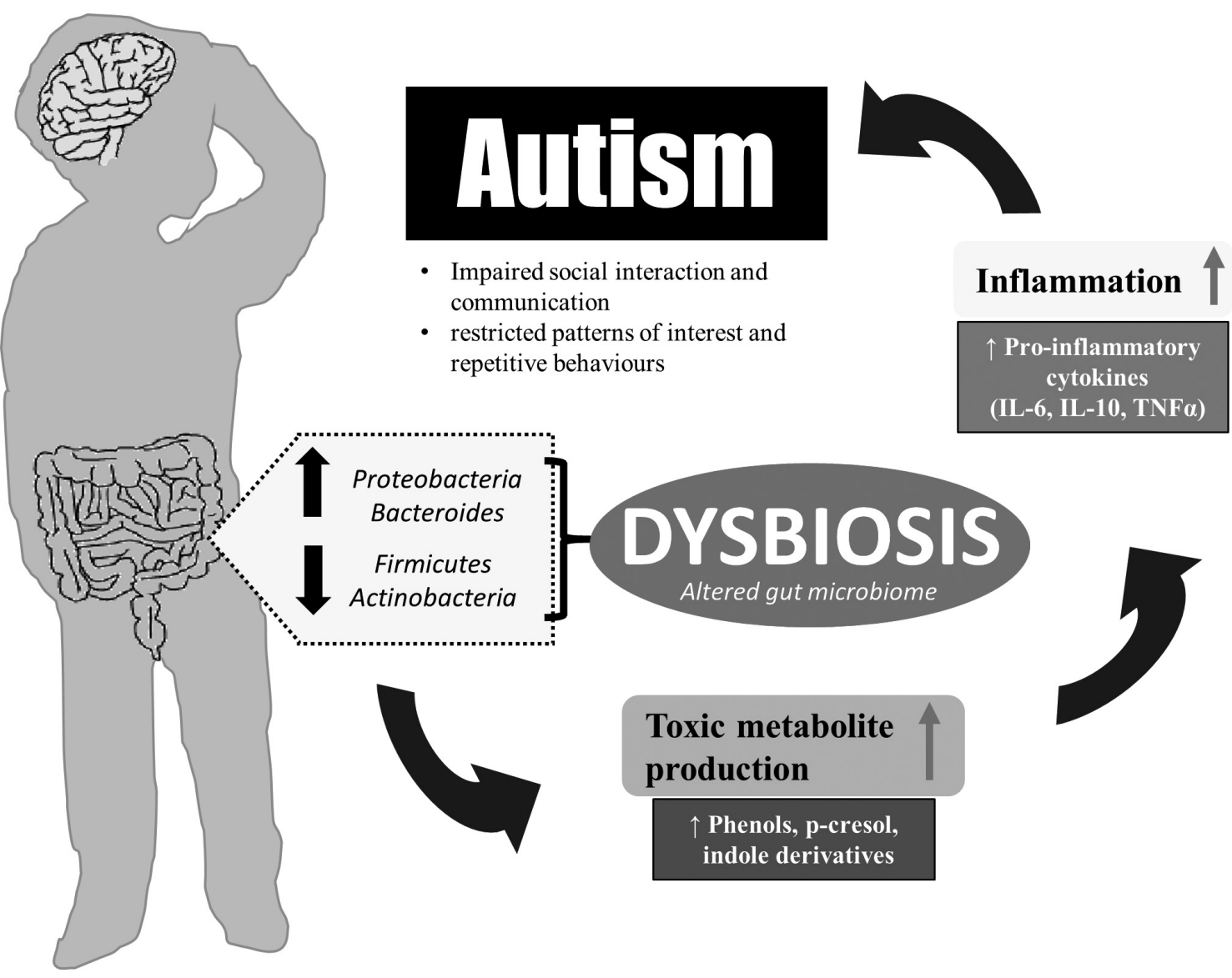

Abstract IDDF2019-ABS-0321 Figure 1 Three studies indicated that there is higher abundance of proteobacteria and bacteroides in autistic children with reduced population of firmicutes and actinobacteria

check, these efforts could improve intestinal and mental health, easing and reducing autistic behaviour in children.

\section{IDDF2019-ABS-0322 DISSECTING THE GUT AND SKIN: BUDDING ASSOCIATION BETWEEN GUT MICROBIOME IN THE DEVELOPMENT TO PSORIASIS?}

${ }^{1}$ Learn-Han Lee*, ${ }^{1}$ Vengadesh Letchumanan, ${ }^{2}$ Tahir Mehmood Khan, ${ }^{3}$ Kok-Gan Chan, ${ }^{4}$ BeyHing Goh, ${ }^{5}$ Nurul-Syakima Ab Mutalib. ${ }^{1}$ Novel Bacteria and Drug Discovery (NBDD) Research Group, Microbiome and Bioresource Research Strength, Jeffrey Cheah School of Medicine and Health Sciences, Monash University Malaysia, Malaysia; ${ }^{2}$ The Institute of Pharmaceutical Sciences (IPS), University of Veterinary and Animal Sciences (UVAS), Pakistan; ${ }^{3}$ Division of Genetics and Molecular Biology, Institute of Biological Sciences, Faculty of Science, University of Malaya, Malaysia; ${ }^{4}$ Biofunctional Molecule Exploratory (BMEX) Research Group, School of Pharmacy, Monash University Malaysia, Malaysia; ${ }^{5}$ UKM Medical Molecular Biology Institute (UMBI), UKM Medical Centre, University Kebangsaan Malaysia, Malaysia

\subsection{6/gutjnl-2019-IDDFabstracts.77}

Background Microbiome consists of normal bacteria flora that resides in the human gut and are key contributors to host metabolism. This symbiotic bond evolves throughout our entire life, from birth to old age, and is the result of different environmental influences. Recently, reports revealed dysbiosis of gut microbiome could relate to psoriasis occurrence. Psoriasis - chronic immune-mediated inflammatory disease affects around $2-4 \%$ of the global population. This systematic review aims to reveal the dysbiosis of the gut microbiome and the occurrence of psoriasis.
Methods A thorough search was conducted using predefined $\mathrm{MeSH}$ terms 'gut', 'microbiome' or 'microbiota' and 'psoriasis' in 3 databases (Pubmed, Medline, ScienceDirect; from database inception to December 2018). All titles and abstracts obtained were screened based inclusion and exclusion criteria. Studies reporting gut microbiome data in relation to gut microbiome effects were included. Studies without gut microbiome data and/or psoriasis were excluded along with conference abstracts, reviews, systematic reviews, meta-analyses, and comments.

Results The gut microbiome is able to control the imiquimod-induced skin inflammation by altering the $T$ cell response, thus affects the pathogenesis of psoriasis. Studies clearly reported the dysbiosis microbiome of psoriasis patients compared to healthy persons. Psoriatic gut microbiome in a few studies revealed an increase presence of Faecalibacterium and a decrease of Bacteroides. Another study reported a high abundance of Bacteroidia in the microbiome of its psoriasis patients, suggesting the potential pathogen that promotes the pathogenesis of psoriasis. As one of the common normal intestinal bacteria in healthy individuals, lower levels of Faecalibacterium prausinitzii have been observed in patients with Crohn's disease, obesity, metabolic syndrome and major depressive diseases. However, in psoriatic patients, Faecalibacterium prausinitzii have been abundant in the gut microbiota - a similar pattern seen in infants with eczema and atopic dermatitis. Probiotics containing normal flora is essential for individuals to maintain a healthy gut microbiota. 